[14] X. Zhang and H. Iwakura, "Design of FIR Nyquist filters using Remez exchange algorithm," IEICE Trans., vol. J79-A, no. 8, pp. 1378-1384, Aug. 1996.

[15] K. Nakayama and T. Mizukami, "A new IIR Nyquist filter with zero intersymbol interference and its frequency response approximation," IEEE Trans. Circuits Syst., vol. CAS-29, pp. 23-34, Jan. 1982.

[16] K. Nakayama and T. Seki, "A design method for multirate filters," Proc. IEEE, vol. 84, pp. 11.8.1-4, 1984

[17] T. Kida and Y. Fukuda, "Design of digital Nyquist filters based on the measure of squared error at sampling points," IEICE Trans., vol. J69-A, no. 3, pp. 359-366, Mar. 1986.

[18] T. Takebe, and T. Matsumoto, "Design of IIR Nyquist filter pairs with equiripple stopband attenuation," IEICE Trans., vol. J69-A, no. 5, pp. 592-599, May 1986.

[19] M. Renfors and T. Saramaki, "Recursive $N$ th-band digital filters-Part I: Design and properties," IEEE Trans. Circuits Syst., vol. CAS-34, pp. 24-39, Jan. 1987.

[20] _ _ "Recursive $N$ th-band digital filters-Part II: Design of multistage decimators and interpolators," IEEE Trans. Circuits Syst., vol. CAS-34, pp. 40-51, Jan. 1987.

[21] T. E. Tuncer and T. Q. Nguyen, "Interpolated IIR $M$ th-band filter design with allpass subfilters," IEEE Trans. Signal Processing, vol. 43, pp. 1986-1990, Aug. 1995.

[22] X. Zhang and H. Iwakura, "Design of IIR Nyquist filters with zero intersymbol interference," IEICE Trans. Fundamentals, vol. E79-A, no. 8, pp. 1139-1144, Aug. 1996

\section{Repeated Filtering in Consecutive Fractional Fourier Domains and Its Application to Signal Restoration}

\author{
M. Fatih Erden, M. Alper Kutay, and Haldun M. Ozaktas
}

\begin{abstract}
Filtering in a single time domain or in a single frequency domain has recently been generalized to filtering in a single fractional Fourier domain. In this correspondence, we further generalize this to repeated filtering in consecutive fractional Fourier domains and discuss its applications to signal restoration through an illustrative example.
\end{abstract}

\section{INTRODUCTION}

Fig. 1(a) shows multiplication of an input signal $f_{\text {in }}(u)$ with a multiplicative filter $h(u)$ to obtain the output signal $f_{\text {out }}(u)$. We call this operation multiplicative filtering in the time domain. Similarly, we refer to the operation in Fig. 1(b) as multiplicative filtering in the frequency domain (note that we are using the same dummy variable $u$ in both the time and frequency domains). In Fig. 1(c), we show multiplicative filtering in the ath-order fractional Fourier transform domain. In this configuration, first the $a$ th fractional Fourier transform of the input is obtained, and then, a multiplicative filter $h(u)$ is applied in this domain. Finally, the resulting waveform is transformed with order $-a$ in order to obtain the output profile in the time domain (the $-a$ th transform is the inverse of the $+a$ th transform). The ath-order fractional Fourier transformation reduces to the identity

Manuscript received May 28, 1997; revised August 25, 1998. The associate editor coordinating the review of this paper and approving it for publication was Prof. Moeness Amin.

M. F. Erden is with Massana Ltd., Dublin, Ireland.

M. A. Kutay is with the Department of Electrical and Computer Engineering, Drexel University, Philadelphia, PA 19104 USA.

H. M. Ozaktas is with the Department of Electrical Engineering, Bilkent University, Ankara, Turkey.

Publisher Item Identifier S 1053-587X(99)03268-7.

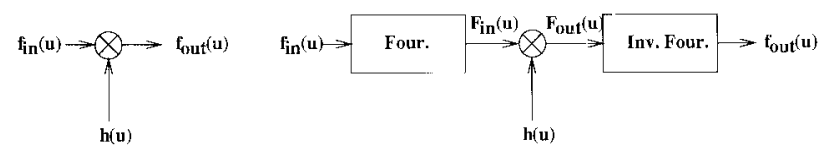

(a)

(b)

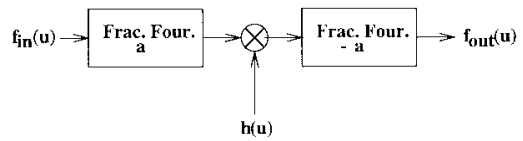

(c)

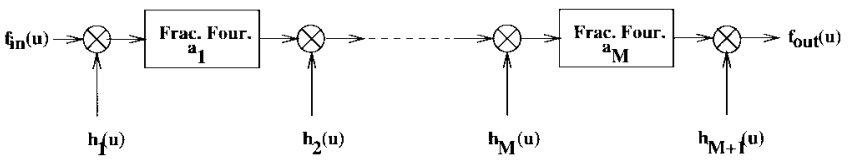

(d)

Fig. 1. Configurations that correspond to (a) filtering in the time domain, (b) filtering in the frequency domain, (c) filtering in a single fractional Fourier domain, (d) repeated filtering in consecutive fractional Fourier domains.

operation for $a=0$; therefore, Fig. 1(c) reduces to Fig. 1(a) for $a=0$. Likewise, it reduces to the ordinary Fourier transformation for $a=1$ so that in this case, Fig. 1(c) reduces to Fig. 1(b). In [1]-[4] it is shown that the added degree of freedom offered by the order parameter $a$ allows improved performance (e.g., smaller meansquare error) in a variety of circumstances including restoration of time-varying signals degraded by nonstationary noise. Furthermore, since both the digital [5] and optical [6]-[8] implementations of the fractional Fourier transformation do not imply extra work compared with the ordinary Fourier transformation, these improvements are achieved at no additional cost.

We can further generalize the concept of single fractional Fourier domain filtering [Fig. 1(c)] to repeated filtering in consecutive fractional Fourier domains [Fig. 1(d)]. Here, we apply the first filter in the 0th fractional domain (the time domain), the second filter in the $a_{1}$ st fractional domain, the third filter in the $\left(a_{1}+a_{2}\right)$ nd fractional domain, and so on. This generalization was first mentioned in [1] and [8]. However, in those papers, the problem of finding the filter profiles for a specified application has not been addressed. In this correspondence, we discuss the applications of this filtering configuration to signal restoration. More specifically, we seek the optimal filter profiles resulting in the minimum mean-square estimate of the original signal.

\section{Fractional Fourier TRANSFORMATION}

The $a$ th-order fractional Fourier transform $p_{a}(u)$ of $p(u)$ is defined for $0<|a|<2$ as

$$
\begin{aligned}
p_{a}(u)= & \sqrt{1-j \cot \phi} \int_{-\infty}^{\infty} \exp \left[j \pi \left(\cot \phi u^{2}\right.\right. \\
& \left.\left.-2 \csc \phi u u^{\prime}+\cot \phi u^{\prime 2}\right)\right] p\left(u^{\prime}\right) d u^{\prime}
\end{aligned}
$$

where $\phi=a \pi / 2$. The kernel is defined separately for $a=0$ and $a= \pm 2$ as $B_{0}\left(u, u^{\prime}\right) \equiv \delta\left(u-u^{\prime}\right)$ and $B_{ \pm 2}\left(u, u^{\prime}\right) \equiv \delta\left(u+u^{\prime}\right)$, respectively [9]. The definition is easily extended outside the interval $[-2,2]$ through $\mathcal{F}^{4 i+a} \hat{q}=\mathcal{F}^{a} \hat{q}$ for any integer $i$. 


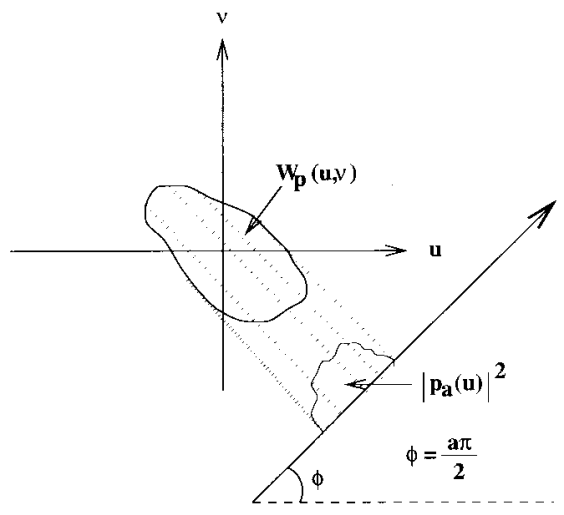

Fig. 2. Relationship between the Wigner distribution and the fractional Fourier transformation of a signal.

Some essential properties of the transformation are the following.

1) It is linear.

2) The first order transformation $(a=1)$ corresponds to the ordinary Fourier transformation.

3) It is additive in index $\mathcal{F}^{a_{1}} \mathcal{F}^{a_{2}} \hat{q}=\mathcal{F}^{a_{1}+a_{2}} \hat{q}$.

Others may be found in [1], [6], and [8]-[10].

There is a close relationship between the fractional Fourier transformation and the Wigner distribution, which is defined as

$$
W_{p}(u, \nu)=\int p\left(u+u^{\prime} / 2\right) p^{*}\left(u-u^{\prime} / 2\right) \exp \left(-j 2 \pi u^{\prime} \nu\right) d u^{\prime} .
$$

The Wigner distribution of $p_{a}(\cdot)$ is simply a rotated form of the Wigner distribution of $p(\cdot)$. Alternatively, we can also relate $W_{p}(\cdot, \cdot)$ directly with $p_{a}(\cdot)$ as [1]

$$
\left|p_{a}(u)\right|^{2}=\mathcal{R}_{\phi}\left[W_{p}(u, \nu)\right]
$$

where $\phi=a \pi / 2$, and $\mathcal{R}_{\phi}[\cdot]$ is the Radon transform evaluated at angle $\phi$. Thus, from (3), we see that the integral projection of the Wigner distribution of $p(\cdot)$ on an axis making an angle $\phi$ with the $u$ axis corresponds to $\left|p_{a}(\cdot)\right|^{2}$ (see Fig. 2).

\section{RePEATED Filtering In Consecutive Domains}

First, let us consider a signal with an additive distortion such that their ordinary Fourier transforms do not overlap. Such a distortion is easily eliminated in the frequency ( $a=1 \mathrm{st}$ ) domain by using a suitable multiplicative filter. For the second example, consider a signal and an additive distortion that do not overlap in the time $(a=0$ th) domain. In this case, we can eliminate the distortion with a suitable filter in the $a=0$ th domain.

Let us now consider the case illustrated in Fig. 3(a), where the Wigner distributions of a desired signal, and undesired noise term, is shown on the same plot. As the projections of these Wigner distributions on both the $u$ and $\nu$ axes overlap, we cannot totally eliminate the effect of the noise term by simply filtering in the $a=0$ th and $a=1$ st domains. However, we can eliminate the noise term completely by filtering in a rotated coordinate system. Thus, we may improve the performance by filtering in a fractional domain, compared with filtering in the time or frequency domains.

We will finally consider the case in Fig. 3(b). This time, the Wigner distributions of the noise term and the desired signal are in such a shape that we cannot find a single rotated coordinate system where we can completely eliminate the noise term from the signal. However, if we consecutively rotate the coordinate system by angles $\phi_{1}, \phi_{2}$, and $\phi_{3}$ and apply suitable multiplicative filters in these coordinate systems, we can completely get rid of the noise term. Thus, for this

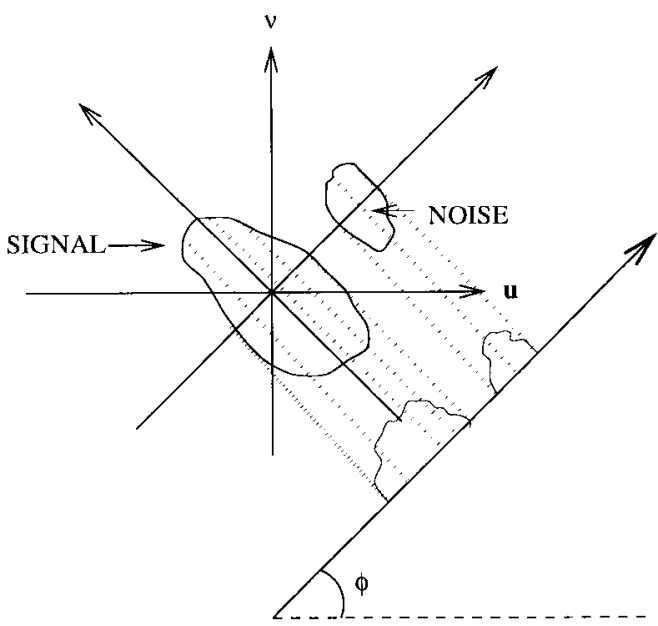

(a)

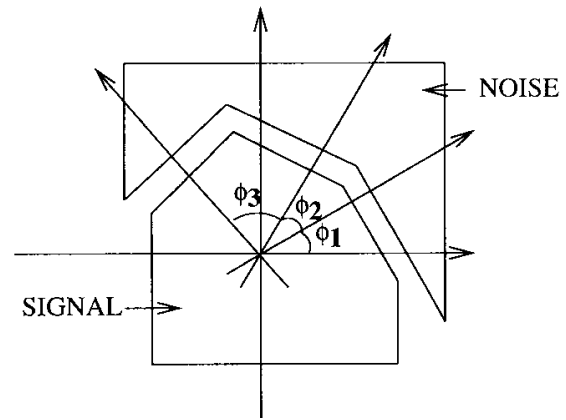

(b)

Fig. 3. Noise separation. (a) In a single fractional Fourier domain. (b) In consecutive fractional Fourier domains.

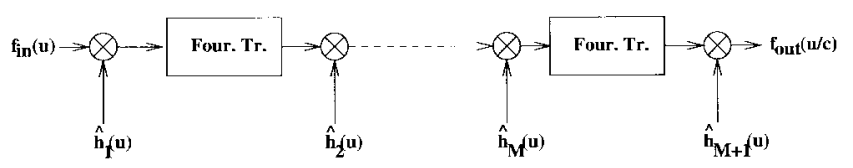

Fig. 4. Canonical form.

specific example, we can completely eliminate the noise term from the desired signal by repeated filtering in consecutive fractional Fourier domains. Single domain filtering is not sufficient.

In Fig. 3(b), we considered the case where the Wigner distributions of the noise and the signal do not overlap in the time-frequency plane. In cases where the Wigner distributions overlap, the filters may be chosen in order to minimize the mean-square error.

Fractional Fourier transformation is relatively easy to visualize by virtue of the fact that the $a$ th fractional domain makes an angle $\phi=$ $a \pi / 2$ with the $u$ axis. However, fractional Fourier transformations are in fact a subclass of linear canonical transformations [11]. Since these transformations can also be implemented in $O(N \log N)$ time, further generality and improvements may be obtained through the use of these transformations. (A single-stage linear canonical transform filtering was treated in [12].) In [15], we have formulated the repeated filtering problem in terms of linear canonical transformations and showed that it can be reduced into an equivalent but simpler form involving only ordinary Fourier transforms (See Fig. 4 for the simple canonical form of the configuration in Fig. 1(d). A weaker form of this result was previously stated in [13] and [14]). There, the discrete version of this problem has also been provided. Again, in [15], the repeated filtering problem has been proposed to synthesize a desired 


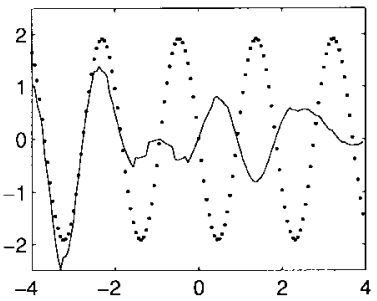

(a)

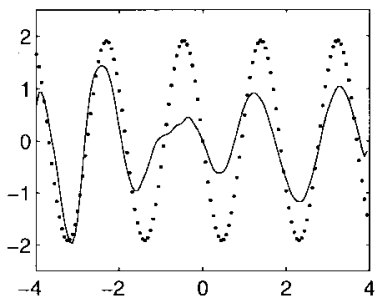

(c)

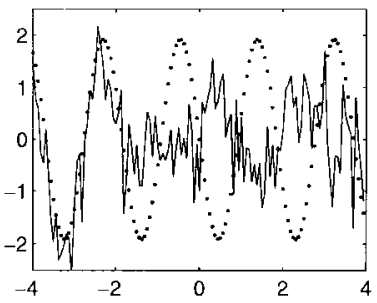

(e)

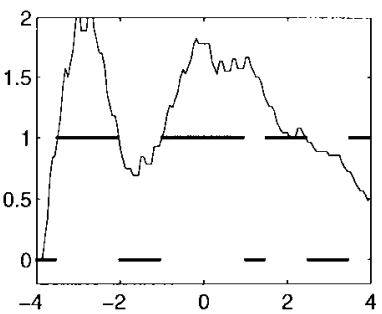

(g)

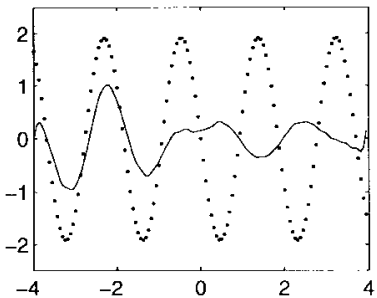

(b)

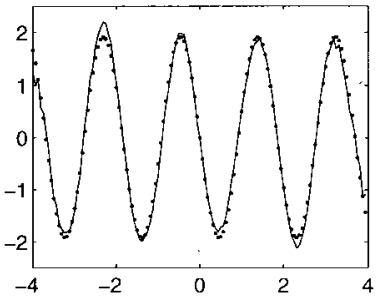

(d)

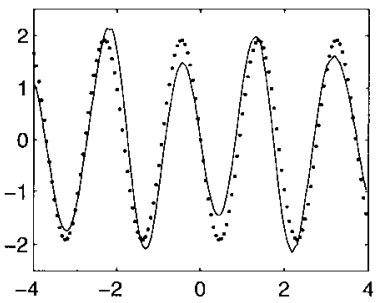

(f)

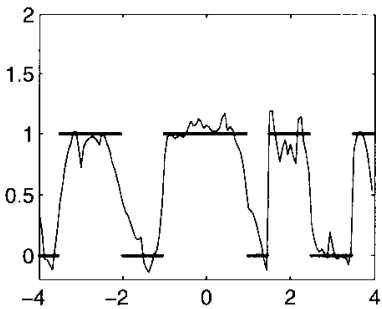

(h)
Fig. 5. (a) Realization of the input process (dotted) and its corresponding degraded output when there is no additive distortion (solid). (b) Estimate obtained by the optimum filter in the ordinary Fourier domain (solid) corresponding to the realization in part (a) (dotted). (c) Estimate obtained by the optimum filter in the optimum fractional Fourier domain (solid). (d) Estimate obtained by repeated filtering in two consecutive domains (solid). (e) Same input realization in part (a) (dotted) and its corresponding degraded output in the presence of additive distortion (solid). (f) Estimate obtained by repeated filtering in four consecutive domains (solid) corresponding to the realization in part (e) (dotted). (g) Typical realization of a pulse train composed of 16 pulses (dotted), and its corresponding degraded output when there is no additive distortion (solid). (h) Estimate obtained by repeated filtering in three consecutive domains (solid) corresponding to the realization in part $(\mathrm{g})$ (dotted).

linear transformation. In this correspondence, we will discuss this problem in terms of signal restoration.

\section{Signal Restoration}

Sometimes, we may want to restore a desired signal that is degraded by a known system and/or by a noise term. With this aim in mind, we search for an appropriate operator that minimizes the effect of degradation and noise. However, this operator strongly depends on the observation model, the design criteria used, and the prior knowledge available about the desired signal and noise. Here, we will assume that we only know the correlation functions of the desired signal and the noise, we will consider the frequently used meansquare error (MSE) criteria, and we will use the observation model expressed as $\bar{y}=\hat{H} \bar{x}+\bar{n}$. Here, $\bar{y}, \bar{x}$, and $\bar{n}$ are the column vectors representing the output, input, and noise processes respectively, and $\hat{H}$ is the matrix characterizing the degradation process. We can now state the problem of finding the optimum linear estimation operator $\hat{G}_{\text {opt }}$, which is defined as

$$
\bar{x}_{e}=\hat{G}_{\mathrm{opt}} \bar{y}
$$

such that the estimate $\bar{x}_{e}$ of $\bar{x}$ minimizes the mean-square error (MSE).

For a time-invariant degradation model $\hat{H}$ with stationary processes $\bar{x}$ and $\bar{n}$, the optimum linear estimation operator corresponds to the classical optimum Wiener filter [16], which is an operation of the form depicted in Fig. 1(b). However, for an arbitrary degradation model or nonstationary processes, the optimum linear estimation operator may not, in general, correspond to a single multiplicative filter in the ordinary Fourier domain. In [2], the optimum Wiener filter is generalized to fractional Fourier domains by using the configuration in Fig. 1(c). In this correspondence, we further generalize by considering repeated filtering configurations. We show that satisfactory results can be obtained in a variety of applications with a moderate number of filters.

\section{A. Mathematical Definition of the Problem}

In this correspondence, input and output processes and noise are considered to be finite-length random processes with size $N$, and we assume that we know the correlation matrices $\hat{R}_{x x}$ and $\hat{R}_{n n}$ of the desired input signal and the noise, respectively. We will further assume that the noise is independent of the input $\bar{x}$, and it has zero mean. Under these assumptions, for the known degradation model, we can also calculate the correlation matrices $\hat{R}_{x y}=E\left[\bar{x} \bar{y}^{H}\right]$ and $\hat{R}_{y y}=E\left[\bar{y} \bar{y}^{H}\right]$. The problem is to obtain the best linear prediction $\bar{x}_{e}$ of $\bar{x}$ that minimizes the mean-square error (MSE), which is defined as

$$
\sigma^{2}=\frac{1}{N} E\left[\left(\bar{x}-\bar{x}_{e}\right)^{H}\left(\bar{x}-\bar{x}_{e}\right)\right]
$$

In [2], the form of the estimate has been restricted to a single filter sandwiched between two fractional Fourier stages. Here, we generalize its form to repeated filtering in consecutive fractional Fourier domains. As we have shown, the equivalence of repeated filtering in consecutive domains with repeated filtering in consecutive ordinary time and frequency domains in [15], an estimate of the form

$$
\bar{x}_{e}=\hat{\Lambda}_{M+1} \hat{F} \cdots \hat{F} \hat{\Lambda}_{k} \hat{F} \cdots \hat{F} \hat{\Lambda}_{1} \bar{y}
$$

is general enough to consider all repeated filtering cases. In this expression, $\hat{\Lambda}_{k}$ is an $N \times N$ diagonal matrix with its diagonal elements equal to that of $\bar{h}_{k}$, and $\hat{F}$ is an $N \times N$ discretetime Fourier transform matrix. Thus, the problem can be restated to obtain the diagonal matrices $\hat{\Lambda}_{M+1}, \hat{\Lambda}_{M}, \ldots, \hat{\Lambda}_{2}, \hat{\Lambda}_{1}$ (or the filters $\left.\bar{h}_{M+1}, \bar{h}_{M}, \ldots, \bar{h}_{2}, \bar{h}_{1}\right)$ in (6) in order to minimize the error expression in (5).

The form of (6) has $(M+1) \times N$ degrees of freedom that is less than the $N^{2}$ degrees of freedom that $\hat{G}_{\text {opt }}$ has in (4). (Here, we are restricting ourselves to moderate values of $M<N$.) The estimator $\hat{G}_{\text {opt }}$ is well known. However, applications of this estimator requires $O\left(N^{2}\right)$ computation time. In contrast, the estimator given in (6) requires $O(M N \log N)$ time. If we can obtain satisfactory estimates using (6), as we showed to be the case in a variety of applications, a considerable savings in time of computation is achieved. 


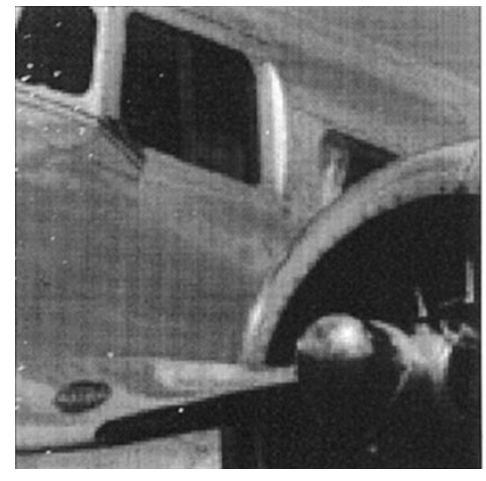

(a)

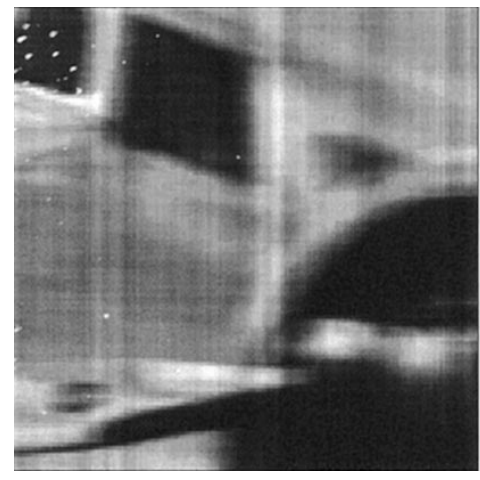

(c)

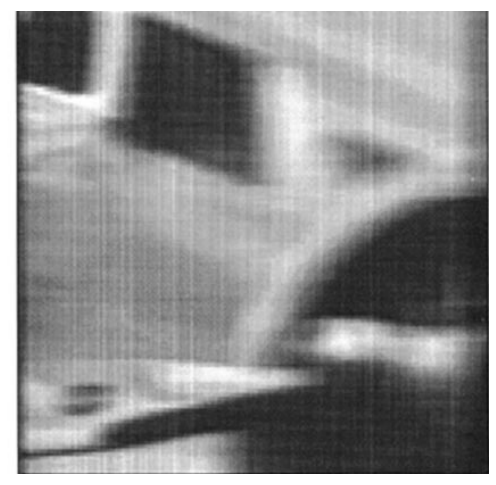

(b)

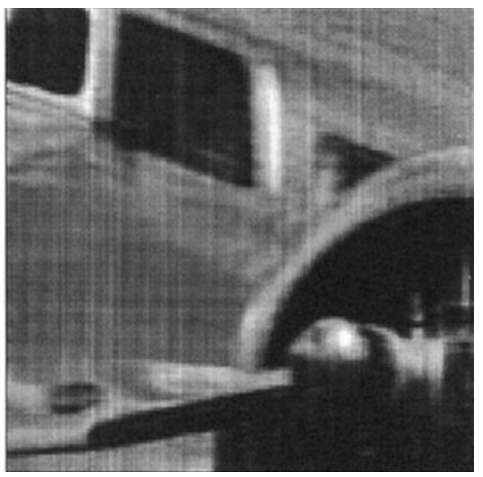

(d)

Fig. 6. (a) Original image. (b) Distorted image obtained by degrading each row of the original image by nonconstant velocity motion blur. (c) Optimum recovered image obtained by single fractional Fourier domain filtering. (d) Recovered image obtained by repeated filtering in four consecutive domains.

\section{B. Solution of the Problem}

We see from (6) that $\bar{x}_{e}$ depends on the filters in a highly nonlinear manner. Thus, we cannot solve for the filters that minimize the error in (5). For this reason, we try an iterative algorithm. In the iteration, we first initialize all the filters. Then, starting with the first filter, we assume that all the filter profiles apart from $\bar{h}_{k}$ are known, and we calculate the optimum expression for the $k$ th filter in terms of the remaining filters. We then go on to $(k+1)$ th filter. When we reach $k=M+1$ and obtain the optimum profile for $\bar{h}_{M+1}$, we set $k=1$ and start again with the first filter. We continue this until the iteration converges.

An important step of the algorithm is to calculate the optimum $k$ th filter in terms of the other filters. Defining $\hat{A}$ and $\hat{B}$ as

$$
\hat{A}=\hat{\Lambda}_{M+1} \hat{F} \cdots \hat{F} \hat{\Lambda}_{k+1} \hat{F}, \quad \hat{B}=\hat{F} \hat{\Lambda}_{k-1} \hat{F} \cdots \hat{F} \hat{\Lambda}_{1}
$$

we can express $\bar{x}_{e}$ to be $\bar{x}_{e}=\hat{A} \hat{\Lambda}_{k} \hat{B} \bar{y}$. We now show how to find the filter $\bar{h}_{k}$ (whose elements are the diagonal elements of $\hat{\Lambda}_{k}$ ) that minimizes the error defined in (5). We write the $m$ th element of the $k$ th filter in terms of its real and imaginary parts as $h_{k m}=h_{k m}^{r}+j h_{k m}^{i}$. We want $\sigma^{2}$ to satisfy

$$
\frac{\delta \sigma^{2}}{\delta h_{k m}^{r}}=0 \quad \frac{\delta \sigma^{2}}{\delta h_{k m}^{i}}=0, \quad m=1,2, \ldots, N .
$$

Starting with the definition of $\sigma^{2}$ in (5), it is possible to show that

$$
\hat{D} \bar{h}_{k}=\bar{c} \text {. }
$$

In this equation

$$
\hat{D}=\left(\hat{A}^{H} \hat{A}\right) \otimes\left(\hat{B} \hat{R}_{y y} \hat{B}^{H}\right)^{T}
$$

where the operator $\otimes$ corresponds to elementwise multiplication of two matrices, and

$$
\bar{c}=\operatorname{diag}\left(\hat{A}^{H} \hat{R}_{x y} \hat{B}^{H}\right)
$$

where $\operatorname{diag}(\cdot)$ is the operator that forms a vector from the diagonal elements of its input square matrix. Thus, as is evident from (9), we have $N$ linear equations corresponding to $N$ unknowns from which we can solve for the coefficients of $\bar{h}_{k}$.

The proposed iterative algorithm always converges to a minimum point. However, because of the nonlinear nature of the problem, the point to which the iteration converges may not and, in general, will not be the global minimum point but rather will be one of the local minima. We ran the algorithm with several initial starting points and picked the one resulting in the smallest mean-square error. We did not overly concern ourselves with determining the global minimum since the values we obtained already represented satisfactory mean-square errors.

\section{An Illustrative Example}

In this subsection, we will apply repeated filtering method to the removal of space-variant blur. The kernel is given by

$$
h\left(x, x^{\prime}\right)=\frac{1}{\alpha x+\alpha_{0}} \operatorname{rect}\left(\frac{x-x^{\prime}}{\alpha x+\alpha_{0}}-\frac{1}{2}\right)
$$

where $\alpha$ and $\alpha_{0}$ correspond to acceleration and initial velocity, respectively.

We will first try the sinusoidal type input process with no additive noise term [see Fig. 5(a)]. For this case, we calculate the MSE values of the optimum filter in the ordinary Fourier domain, the optimum filter profile as proposed in [2], and our repeated filtering method with three filters $(M=3)$ to be $2.07,1.80$, and 0.12 , respectively. Thus, we observe a considerable decrease in MSE, where we can also visualize this in Fig. 5(b) to Fig. 5(d). However, for this case, the performance of the optimum linear estimator is much better than the one corresponding to the repeated filtering method with three 
filters. Nevertheless, by increasing the number of filters $M$, we can increase the performance. For example, we obtain MSE around 0.04 when $M=5$.

We later considered the degradation system with an additive white Gaussian noise whose energy corresponds to SNR $=5$ [see Fig. 5(e)]. For this case, the MSE values were 2.68 for the optimum filter in the ordinary Fourier domain, 2.17 for the optimum filter proposed in [2], and 0.16 for our repeated filtering method with $M=5$. The estimate of our repeated filtering method is illustrated in Fig. 5(f). Moreover, the MSE value we obtain with five filters is comparable with the one that belongs to the optimum linear estimator, which is around 0.13 .

Now, let us consider pulse-code modulator (PCM) like signals, each of which consists of 16 pulses of value either 0 or 1 . This can also represent a common bar code. Here, we assume that there is no additive distortion [see Fig. 5(g)]. The corresponding repeated filtering output for a typical realization of the input is illustrated in Fig. 5(h). Here, $M=4$, and the corresponding MSE value is 0.05 . The performance of the optimum linear estimator again comes out to be much better than the repeated filtering method with $M=4$. However, as we see in Fig. 5(h), the binary values of the pulses can be determined with the help of a comparator, which compares the values of the actual output with 0.5 (Thus, the repeated filtering with $M=4$ together with a comparator may be sufficient. If greater accuracy is needed, we may increase $M$ to five, in which case, the MSE turns out to be $4 \times 10^{-6}$.

Finally, we consider the effect of the nonconstant velocity motion blur on the whole image in Fig. 6(a). The distorted image is shown in Fig. 6(b). In Fig. 6(c), we showed the result of the optimal filtering in a single domain. The MSE value for this case is 0.10 . Finally, the result obtained with five repeated filters is shown in Fig. 6(d), where the MSE value is 0.03 . We can also visualize the differences in MSE values by looking at the corresponding images in Fig. 6. For this specific input process, the MSE value of the optimum linear estimator is $1.2 \times 10^{-5}$.

\section{CONCLUSION}

In this correspondence, we generalize the concept of single fractional-Fourier-domain filtering to repeated filtering in consecutive fractional Fourier domains. The repeated filtering problem has also been considered in [15], and it has been applied to the synthesis of desired linear transformations. Here, we consider the repeated filtering configuration in conjunction with signal restoration, and we give an illustrative example to compare its performance with the existing methods. (More examples of the repeated filtering method on signal restoration can be found in [17].) As a result of this comparison, we see that the repeated filtering method we proposed offers better performance than single domain filtering methods with little increase in cost and much lower cost than general linear estimation with performance, which may either be acceptable in certain circumstances or even close to optimal. Thus, the method lies between single domain filtering methods and the general linear estimation method in terms of both cost and performance. Recently, the concept of repeated filtering has been generalized to include more general configurations (e.g., see [18]-[20]).

\section{REFERENCES}

[1] H. M. Ozaktas, B. Barshan, D. Mendlovic, and L. Onural, "Convolution, filtering, and multiplexing in fractional Fourier domains and their relation to chirp and wavelet transforms," J. Opt. Soc. Amer. A., vol. 11, pp. 547-559, 1994.

[2] M. A. Kutay, H. M. Ozaktas, O. Arikan, and L. Onural, "Optimal filtering in fractional Fourier domains," IEEE Trans. Signal Processing, vol. 45, pp. 1129-1143, May 1997.
[3] Z. Zalevsky and D. Mendlovic, "Fractional Wiener filter," Appl. Opt., vol. 35, pp. 3930-3936, 1996.

[4] M. F. Erden, H. M. Ozaktas, and D. Mendlovic, "Synthesis of mutual intensity distributions using the fractional Fourier transform," Opt. Commun., vol. 125, pp. 288-301, 1996.

[5] H. M. Ozaktas, O. Arikan, M. A. Kutay, and G. Bozdagi, "Digital computation of the fractional Fourier transform," IEEE Trans. Signal Processing., vol. 44, pp. 2141-2150, Sept. 1996.

[6] A. W. Lohmann, "Image rotation, Wigner rotation, and the fractional order Fourier transform," J. Opt. Soc. Amer. A., vol. 10, pp. 2181-2186, 1993.

[7] H. M. Ozaktas and D. Mendlovic, "Fractional Fourier optics," J. Opt. Soc. Amer. A., vol. 12, pp. 743-751, 1995.

[8] H. M. Ozaktas and D. Mendlovic, "Fractional Fourier transforms and their optical implementation: II," J. Opt. Soc. Amer. A., vol. 10, pp. 2522-2531, 1993.

[9] A. C. McBride and F. H. Kerr, "On Namias's fractional Fourier transform," IMA J. Appl. Math., vol. 39, pp. 159-175, 1987.

[10] L. B. Almeida, "The fractional Fourier transform and time-frequency representations," IEEE Trans. Signal Processing, vol. 42, pp. 3084-3091, 1994.

[11] K. B. Wolf, Integral Transforms in Science and Engineering. New York: Plenum, 1979

[12] B. Barshan, M. A. Kutay, and H. M. Ozaktas, "Optimal filtering with linear canonical transformations," Opt. Commun., vol. 135, pp. 32-36, 1997.

[13] H. M. Ozaktas, "Repeated fractional Fourier domain filtering is equivalent to repeated time and frequency domain filtering," Signal Process., vol. 54, pp. 81-84, 1996.

[14] H. M. Ozaktas and D. Mendlovic, "Every Fourier optical system is equivalent to consecutive fractional Fourier domain filtering," Appl. Opt., vol. 35, pp. 3167-3170, 1996.

[15] M. F. Erden and H. M. Ozaktas, "Synthesis of general linear systems with repeated filtering in consecutive fractional Fourier domains," $J$. Opt. Soc. Amer. A., vol. 15, pp. 1647-1657, 1998.

[16] N. Mohanty, Signal Processing. New York: Van Nostrand Reinhold, 1987.

[17] M. F. Erden, "Repeated filtering in consecutive fractional Fourier domains," Ph.D. dissertation, Bilkent Univ., Ankara, Turkey, 1997.

[18] M. A. Kutay, M. F. Erden, H. M. Ozaktas, O. Arikan, O. Guleryuz, and C. Candan, "Space bandwidth efficient realizations of linear systems," Opt. Lett., vol. 23, pp. 1069-1071, 1998.

[19] M. A. Kutay, M. F. Erden, H. M. Ozaktas, O. Arikan, C. Candan and O. Guleryuz, "Cost-efficient approximation of linear systems with repeated and multichannel filtering configurations," in Proc. IEEE Int. Conf. Acoust., Speech, Signal Process. (ICASSP), Seattle, WA, May 12-15, 1998.

[20] M. A. Kutay, "Generalized filtering configurations with applications in digital and optical signal and image processing," Ph.D. dissertation, Bilkent Univ. Ankara, Turkey, 1999. 